\title{
The Physarum Computer
}

\author{
Kurt Mehlhorn
}

Max-Planck-Institut für Informatik, Saarbrücken, Germany

Physarum is a slime mold. It was observed over the past 10 years that the mold is able to solve shortest path problems and to construct good Steiner networks $[2,4]$. In a nutshell, the shortest path experiment is as follows: A maze is built and the mold is made to cover the entire maze. Food is then provided at two positions $s$ and $t$ and the evolution of the slime is observed. Over time, the slime retracts to the shortest $s$ - $t$-path.

A mathematical model of the slime's dynamic behavior was proposed in [3]. The network is modelled as an undirected graph $G=(V, E)$ with two special vertices $s$ and $t$. Each edge $e$ has a diameter $D_{e}$ and a length $L_{e}$. The length is fixed and the diameter changes over time. A flow of one is sent from $s$ to $t$ according to the following rule. The network is interpreted as an electrical network where the edge $e$ has resistance $L_{e} / D_{e}$. Each vertex $v$ has a potential $p_{v} ; p_{t}=0$. For an edge $e=(u, v)$, we have a flow $Q_{e}=\left(p_{u}-p_{v}\right) D_{e} / L_{e}$. If $Q_{e}<0$, the flow is in the direction from $v$ to $u$. We have flow conservation in all vertices except for $s$ and $t$. The net flow out of $s$ is one and the net flow into $t$ is one. The edge diameters develop according to the rule

$$
\dot{D}_{e}=\left|Q_{e}\right|-D_{e}
$$

where $\dot{D}_{e}$ is the derivative of $D_{e}$ with respect to time. If the current flow is larger (smaller) than the current diameter, the diameter increases (decreases).

Extensive computer simulations confirm the experimental findings. For the edges on the shortest $s$ - $t$-path, the diameter converges to one, and for the edges off the shortest path, the diameter converges to zero.

For some simple graphs, convergence proofs are available. In particular, if $G$ is a planar graph with $s$ and $t$ being on the boundary of the same face, convergence is shown in [1].

We review the work on the Physarum computer.

\section{References}

1. Miyaji, T., Ohnishi, I.: Physarum can solve the shortest path problem on riemannian surface mathematically rigourously. International Journal of Pure and Applied Mathematics 47, 353-369 (2008)

2. Nakagaki, T., Yamada, H., Tóth, Á.: Maze-solving by an amoeboid organism. Nature 407, 470 (2000)

3. Tero, A., Kobayashi, R., Nakagaki, T.: A mathematical model for adaptive transport network in path finding by true slime mold. Journal of Theoretical Biology, 553-564 (2007)

4. Tero, A., Takagi, S., Saigusa, T., Ito, K., Bebber, D., Fricker, M., Yumiki, K., Kobayashi, R., Nakagaki, T.: Rules for biologically inspired adaptive network design. Science 327, 439-442 (2010) 